\title{
Utilization of the Adolescent Friendly Health Services by the Respective Clients in Otjozondjupa Region of Namibia
}

\author{
M. Muyenga ${ }^{1}$, K. Amakali ${ }^{2}$ \& W. Wilkinson ${ }^{2}$ \\ ${ }^{1}$ School of Public Health, Faculty of Health Sciences, University of Namibia, Windhoek, Namibia \\ ${ }^{2}$ School of Nursing, Faculty of Health Sciences, University of Namibia, Windhoek, Namibia \\ Correspondence: Kristofina Amakali, School of Nursing, Faculty of Health Sciences, University of Namibia, \\ Private Bag 13301, Windhoek, Namibia. Tel: 264-61-206-3297. E-mail: kamakali@unam.na
}

Received: May 20, 2017 Accepted: June 23, 2017 Online Published: December 28, 2017

doi:10.5539/gjhs.v10n2p82 URL: https://doi.org/10.5539/gjhs.v10n2p82

\begin{abstract}
Adolescents experience development related challenges more so those related to the reproductive health. If they are not guided during this transitional stage, they may reap detrimental results including teenage pregnancy. Despite the existence of the national standards for Adolescent Friendly Health Services (AFHS) to prevent among others teenage pregnancy, adolescent pregnancy continues to rise across the regions of Namibia.

This article presents the findings of quantitative, descriptive, cross-sectional study regarding the utilization of the Adolescent Active Participation component of the Adolescent Friendly Health Services standards by the adolescent girls in Otjozondjupa region of Namibia.

Quantitative data were collected through structured questionnaire from a sample of 540 out of study population of 4995 school-going teenage girls as the potential users of the AFHS in Otjozondjupa Region.

The findings indicated that, although the standards for the implementation of AFHS exist, adolescent girls do not utilize the services as demonstrated by high percentage $(96 \%)$ of lack of awareness of the existence of the AFHS among the study participants, low (33\%) use of condom among the sexually active study participants, non-participation (92\%) of adolescent girls in health care committees as well as adolescents' lack of participation $(91 \%)$ in development and distributions of the Information Education Communication materials (IEC). Therefore, the study recommended the strategies which may accelerate active participations of the adolescents in the implementation of the AFHS standards, particularly in Otjozondjupa region and Namibia at large.
\end{abstract}

\section{Introduction}

The transition from childhood to adulthood poses risks of teenage pregnancies which often results into devastating psychological and economic consequences for the adolescent concerned and their babies. It is therefore important that special guidance is provided to the adolescents in order to ensure a smooth transition to adulthood (Larke \& Cleophas-Mazige, 2010; Kesterton \& Cabral de Mello, 2010). Consequently, a global consultation on adolescent friendly health services in 2001 recommended member countries to develop the standards for the improvedservices provision to the adolescents (WHO, 2012). The goal for these standards is to ensure that a comprehensive adolescent health service which is offered through a multi-sectorial approach is available and accessible to all the adolescents.

The standards outline six components for a package of essential services for the adolescents at public health facilities in order to prevent among others, teenage pregnancy. These six components include the Adolescent Active Participation, Community Support and Participation, Adolescent Friendly Health Service Providers, Adolescent Friendly Health Environment, Adolescent Friendly Health Procedures as well as Networking and Collaboration (Ministry of Health and Social Services (MoHSS, 2011; WHO, 2002), as elaborated in the article on the implementation of the AFHS by nurses from the same study (Muyenga, Amakali, \& Wilkinson, 2017). This article presents the findings on the utilization of the AFHS initiatives by the adolescent girls as a reflection of the component on adolescent active participation.

The Adolescent Active Participation component calls for active participation of the adolescents in adolescent focused health promotion programmes, while they should be consulted about their health care needs in order to inform the content for adolescent health services. Therefore, the subsequent component on Community Support 
and Participation advocates for the establishment of Health Facility Committees (HFC) of which adolescents should be members in order to maximize their active participation (United Nations Children's Emergency Fund \& Ministry of Health and Social Services, 2008). In return, adolescents'active participation builds adolescents' skills and confidence in decision making on issues that affect them (WHO, 2002).

Despite the implementation of the Adolescent Friendly Health Services standards in Namibia since 2011, Otjozondjupa region ranks $4^{\text {th }}$ out of the 14 regions in teenage pregnancy in Namibia with 120 live births per 1000 women (MoHSS, 2008). In 2008, the average teenage pregnancy in Otjozondjupa region was 5.9\%, and in 2012, after the implementation of the AFHS standards, the average teenage pregnancy was only $5.3 \%$ (MoHSS, 2012). The average difference of $0.6 \%$ between the rates recorded before and after the implementation of the standards in the region is very minimal. Given the implementation of the AFHS standards from 2011, one expects the average difference of teenage pregnancy to be more than $0.6 \%$.

High rate of teenage pregnancy in spite of the implementation of the AFHS standards concurs with a report by the United Nations Population Fund and the United Nation Human Commission for Refugees (2012) who indicate that despite the existence of services for promotion of adolescents' health, there had been little documentation on the efficiency in deliverance and the effectiveness of such services. Therefore, the question to be answered in the current study was: whether teenage girls in the Otjozondjupa region are aware of the existence of the Adolescent Friendly Health Services standards in the region and whether they utilize the services. It was therefore important to assess the knowledge about the Adolescent Friendly Health Services and the utilization of the services by the adolescent girls in Otjozondjupa region as a measure to prevent among other, teenage pregnancy.

\section{Goal and Objectives}

The goal for the study was to determine if the adolescent girls in the Otjozondjupa region utilize the Adolescent Active Participation component of the Adolescent Friendly Health Services.

The study objective was to determine the knowledge and the utilization of the Adolescent Friendly Health Services by the adolescent girls in Otjozondjupa region.

\section{Research Design and Methods}

\subsection{Design}

A cross-sectional quantitative and descriptive design was applied (Brink, van Rensburg, van der Walt, 2012; Babbie, Mouton, 2010).

\subsection{Study Population}

The study and target population was the 4995 school-going teenage girls in Otjozondjupa region.

\subsection{Inclusion and Exclusion Criteria}

All the adolescent girls of age 13-18 years, who attended school at 21 Secondary Schools in Otjozondjupa region at the time of the study, were included in the study. Adolescent girls who did not attend school, despite that they were within the boundaries of the region were excluded from the study. Adolescent boys were also excluded from the study.

\subsection{Sampling and Sample Size}

Using the Epi Info Version 7 to ensure 95\% confidence level, a sample of 540 was secured from the study population of 4996 school going adolescent girls (Directorate Education, Otjozondjupa Regional Council, 2015).

\subsection{Data Collection Tool}

A structured questionnaire was used to collect the data on adolescents' knowledge about and utilization of the Adolescent Friendly Health Services as well as their participation in health facility committees (HFC).

\subsection{Data Collection Methods}

A self-administered questionnaire was completed by the participants under the direct supervision of the researcher.

The participants' knowledge of Adolescent Friendly Health Services and the utilization of the Adolescent Active Participation component of the Adolescent Friendly Health Services were assessed. Completed questionnaires and checklists were numbered and sealed for validation by the researchers before analysis.

\subsection{Data Analysis}

The data were analysed using the SPSS Version 16 data base for descriptive statistics (De Vos, Strydom, Fouche \& Delport, 2011). Descriptive analyses were applied. 


\section{Ethical Implications}

Since this was an empirical study involving humans, ethical principles of justice, autonomy, protection of participants from harm and beneficence was observed. As regards justice or a fair treatment of the study participants, the study was conducted among female school-going adolescents because as potential users of Adolescent Friendly Health Services initiatives, they were well-placed to provide needed information about their participations.

Adolescent participants were protected from harm that could be occasioned by the research activities. In this regard, permission to conduct the research at schools in Otjozondjupa region was obtained from the Regional Director of Education in the region. Written assent to interview the teenage girls were obtained from the teenage girls' parents. The questionnaire did not contain intrusive questions (Joubert\& Ehrlich, 2014). Participation was voluntarily and the participants could withdraw from the study at any time. Confidentiality was maintained at dissemination of the study results (Parahoo, $2006 \&$ Streubert Speziale\& Carpenter, 2007).

The principle of beneficence was observed because the findings could guide the deliverance of Adolescent Friendly Health Friendly Services in the region (Joubert \& Ehrlich, 2007).

\section{Results}

\subsection{Sample Achievement}

The actual study sample yielded above $80 \%$, of the estimated study sample as displayed in Table 1 .

Table 1. Data collection sample achievement

\begin{tabular}{llll}
\hline Study data collection Source & Study estimated sample & Actual achieved sample & Percentage yield \\
\hline Adolescents & 540 & 437 & $81 \%$ \\
\hline
\end{tabular}

\subsection{Sample Characteristics}

The age ranges of the study participants was $13-19$ years, with the mean age of 16 years. The majority (53.1\%) of the study participants were in grades eight and nine. There were relatively few participants of grades 11 and 12 . The findings of age and grades of the participants are displayed in Figure 1.
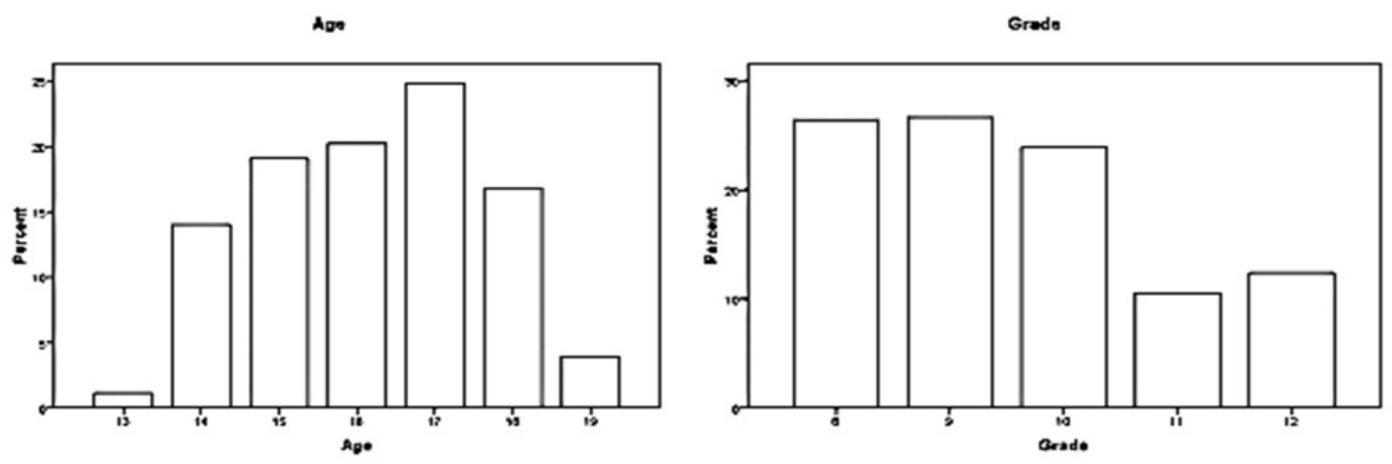

Figure 1. Age and Grade of the adolescent study participants

\subsection{Adolescents' Active Participation}

The study revealed that a higher proportion $(80.8 \%, n=353)$ of the adolescents participants were not aware of the existence of the Adolescent Friendly Health Services. Equally, the majority, $(96.1 \%, n=420)$ of the participants indicated that they do not make use of the adolescent friendly health services. 
Table 2. Knowledge and use of Adolescent Friendly Health Services by the adolescent girls

\begin{tabular}{lllllll}
\hline \multicolumn{2}{l}{ Knowledge on Adolescent Friendly Health Services } & \multicolumn{3}{l}{ Use of Adolescent Friendly Health Services } \\
\hline & Frequency & Percent & & Frequency & Percent \\
\hline $\begin{array}{l}\text { Do not know about } \\
\text { Friendly Health Services }\end{array}$ & Adolescent & 353 & 80.8 & $\begin{array}{l}\text { Do not use the Adolescent Friendly } \\
\text { Health Services }\end{array}$ & 420 & 96.1 \\
$\begin{array}{l}\text { Awareness of the Adolescent Friendly } \\
\text { Health Services }\end{array}$ & 84 & 19.2 & $\begin{array}{l}\text { Use the Adolescent Friendly Health } \\
\text { Services }\end{array}$ & 15 & 3.4 \\
Total & 437 & 100 & Total & 437 & 100 \\
\hline
\end{tabular}

\section{The utilization of Adolescent Friendly Health Services by the adolescent girls}

As the findings showed that very few of the participants were aware of the Adolescent Friendly Health Services, only a limited number $(3.5 \%, \mathrm{n}=15]$ of the study participants reported to have visited the local health facility and made use of the adolescent friend health services standards.

\section{Sexually active and use of condoms among the adolescents}

Out of a sample of 437 adolescent girls, $2 \%(n=9)$ did not respond to the question on whether they were sexually active. A mere $32 \%(n=137)$ out of the remaining 428 respondents who attempted the question, indicated that they were sexually active. Furthermore, out of the 137 respondents to this question, only $24 \%(n=33)$ of them reported to consistently use a condom when having sex. The majority $67 \%(n=92)$ of the adolescents who were sexually active but who did not use condoms, reported to had used other methods of contraceptives. These findings are displayed in Figure 2 and Figure 3 respectively.

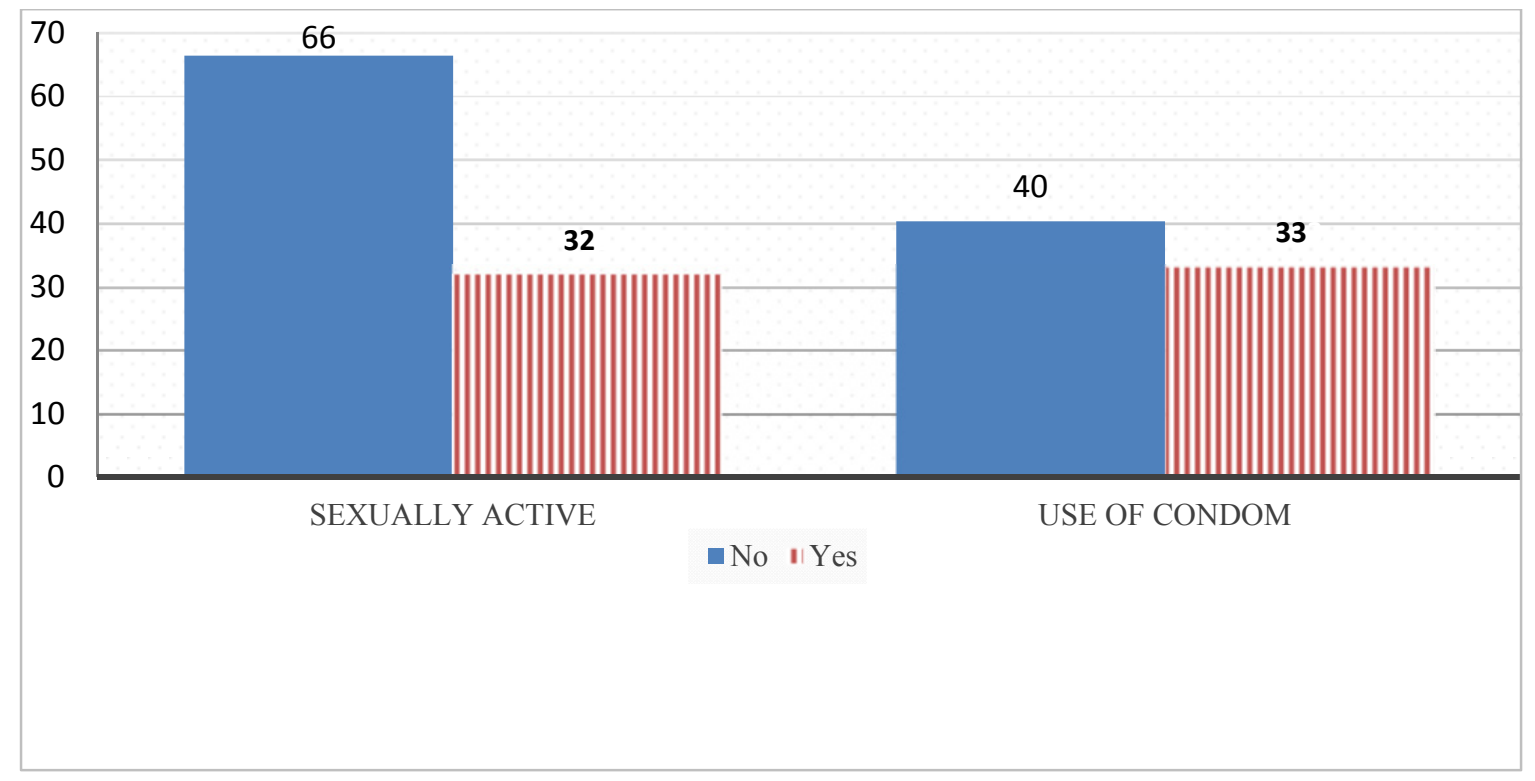

Figure 2. Adolescents sexually active and use of condoms 


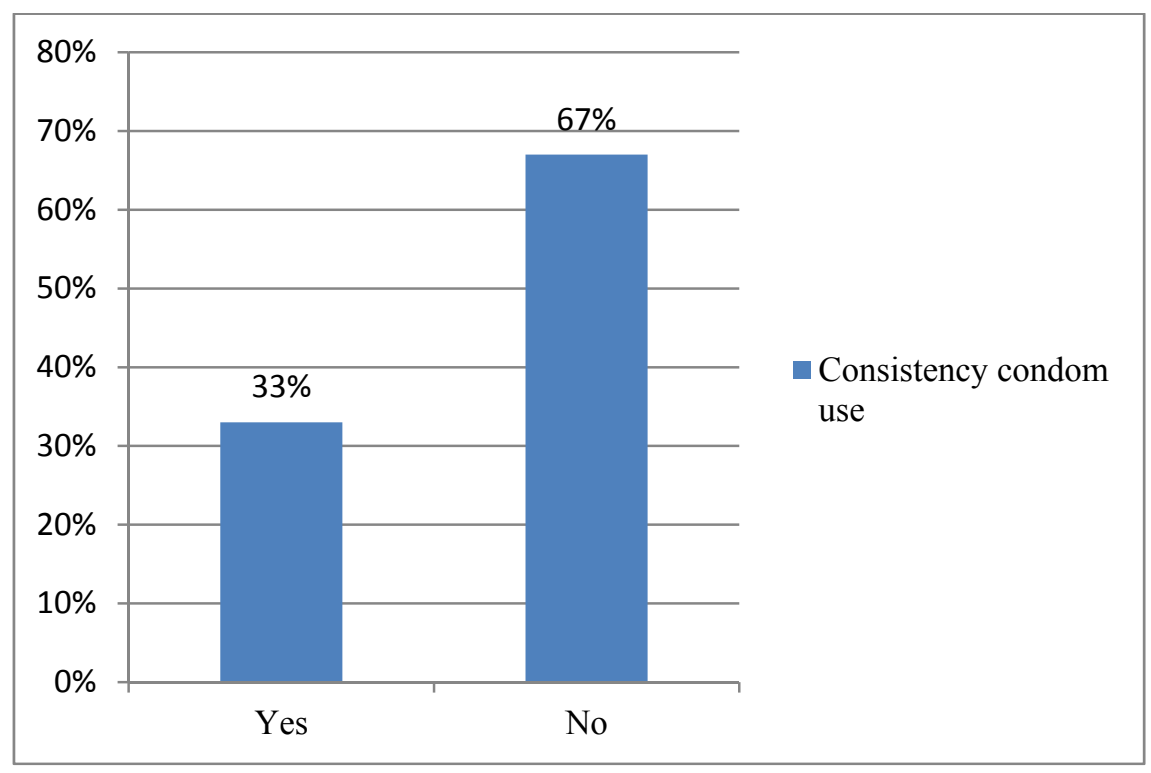

Figure 3. Consistency use of condom

Another variable that was assessed was the adolescents' participation in Health Facility Committee (HFC), and designing and distribution of the Information, Education and Communication (IEC) materials. The findings indicated that a mere $6 \%$ of the study participants knew of their colleagues who were members of the local health facility committee and less than $10 \%$ of the adolescents did participate in designing the development and distribution of the IEC materials and condoms at schools. A mere $20 \%$ of the study participants were involved in conducting health education sessions at schools. Table 3didplays the findings for these variables.

Table 6. Adolescents level of participation in key AFHS activities

\begin{tabular}{llll}
\hline Variables & Responses & Frequency & Percent \\
\hline Any of your colleagues who are members of the Health Facility Committee (HFC)? & No & 402 & 92,0 \\
& Yes & 25 & 5,7 \\
\hline $\begin{array}{l}\text { Are you involved in the design and development of the Information Education and } \\
\text { Communication (IEC) materials? }\end{array}$ & Yes & 397 & 90,8 \\
\hline $\begin{array}{l}\text { Are you or your classmates involved in the distribution of IEC materials and } \\
\text { condoms at school? }\end{array}$ & No & 405 & 6,2 \\
\hline Do you conduct health education sessions among yourselves at school? & No & 20 & 4,6 \\
\hline
\end{tabular}

\subsection{Reliability and Validity}

Reliability of the data was ensured by the pilot testing of the data collecting instrument on 10 teenage girls of similar age and context to that of the study participants in the actual study (De Vos et al., 2011). Face and content validity were ensured. The content for the questionnaire was sufficient to assess all essential information on the utilization of the AFHS by the adolescents. Equally, the questionnaire focused on the adolescents' perspective only. A sample size calculated at $95 \%$ confidence level and simple random sampling of the participants (Babbie \& Mouton, 2001; Brink, 2008; De Vos et al., 2005) also ensured validity of the data. The next sessions provide the discussions on the findings and place them in the context of the existing literature.

\section{Discussion}

\subsection{Discussion}

The purpose of the study was to determine if adolescent girls in Otjozondjupa region utilizes the Adolescent Active 
Participation component of the AFHS standards in order to make inference on the standards' effectiveness in promoting sexual and reproductive health for the respective clients.

The variables that were assessed under this component are: awareness of the AFHS by the adolescents, adolescents' health seeking behaviours-the utilization of the AFHS, adolescents' sexual activities and the use of condom as the inferentially to the knowledge and use of Adolescent Friendly Health Services initiatives by the adolescent girls. Adolescents' participation in the Health Facility Committee (HFC), designing and distribution of Information, Education and Communication (IEC) materials which are also the indicators of adolescent active participation, were equally assessed. The findings are discussed in the next sections.

\section{Awareness of Adolescent Friendly Health Services by the adolescent girls}

Njoroge et al., (2010) postulate that it is a common phenomenon that young people lack the knowledge on sexual matters, in spite of them being sexually active. The findings from this study indicated that a higher proportion $(80 \%)$ of the study participants was not aware of the existence of the Adolescent Friendly Health Services. As a result, only a small proportion of the participants had used the adolescent friendly health services. The cultural context of the study participants regards providing information about sexuality to the adolescents as a taboo. This perception is based on an assumption that providing such information to a teenage would lead to sexual experimentation and sexual promiscuity. By the implication, the nurses as the providers for the AFHS initiatives may also regard access to reproductive health information by the adolescents as a taboo. As a result, they too may have reservations on providing reproductive-based health services to the adolescents. Concurrently, the same study suggested that reproductive health related information to the adolescents seems not have been incorporated in the school outreach programme which is carried out by the nurses in the region. A low display of information on the availability of condoms in the health care facilities (Muyenga, Amakali\& Wilkinson, 2017), also testifies that health care providers at the health care facilities are not pro-adolescent friendly health services.

Furthermore, the same cultural perception may also compromise the teaching for extra-curricular life skills at school [to create awareness of reproductive related issues] as is articulated in the life skill manual under the theme: "my future is my choice" (UNICEF, 2011 a; Directorate Education, Otjozondjupa Regional Council, 2014) because school teachers may share the same cultural sediment. Hence there is a demonstration of lack of awareness among adolescents of the initiative, which is intended to prevent developmental reproductive related health problems, but to promote their well-being.

The findings indicated that there is a need, through a multi-sectoral approach to intensify the effort to create awareness of the AFHS initiatives among the adolescents in Otjozondjupa region. Possession of such information would urge the adolescents to utilize the services and equally guide their sexual behaviours. In the same way, the findings are suggestive of a paradigm shift for the health care workers to reserve their cultural beliefs and dispense necessary reproductive health services to the adolescents including providing reproductive health information for the clients to make informed choices.

\section{Utilization of the Adolescent Friendly Health Services by the adolescent girls}

The results from this study showed a low utilization of the Adolescent Friendly Health Services initiative by the adolescent girls as only a limited number of the study participants reported to have used the adolescent friendly health services. Thus, in contrary to the submissions by the nurses in the same study that school outreach is conducted in the region and health information is provided to learners (Muyenga, Amakali, \& Wilkinson, 2017), the findings call for the sensitization about the availability of adolescent friendly health services initiative and the provision of reproductive health related information, all that would guide the adolescents to make appropriate choices for reproductive health and change their risky sexual behaviours. Equally, health care providers should display an inviting attitude towards the adolescents who seek reproductive services. An inviting attitude of health care providers encourages the adolescents to utilize the services. Another variable that was assessed is the use of condom in relation to being sexual active, findings of which are discussed in the next session.

\section{Sexually active and the use of condoms}

The findings indicated that while the participants were sexually active, only a few of those who are sexually active used condom and even so inconsistently. Low level of the use of condom can be tailored to their low participation in the AFHS initiatives as explained in the preceded section. However, low and inconsistent use of condoms in the era of HIV epidemic is a matter of concern. Sexually active adolescents need to be informed that the use of condom is an essential option for dual protections against pregnancy and STIs including the ruinous HIV infection.

Awareness and utilization of the Adolescent Friendly Health Services by the adolescents is a prerequisite for the adolescents' participation in Health Facility Committee (HFC), designing and distribution of Information, 
Education and Communication (IEC) materials. However, given a lack of awareness and poor utilization of the services, the findings on adolescents' participation in health committees and distribution of health information affirm likewise.

\section{Adolescents' participation in Health Facility Committee (HFC), designing of Information, Education and Communication (IEC) materials}

The study further explored the participation of the adolescents in health facility committees and the designing and distribution of the IEC materials. In addition, adolescents were also asked if they conducted health education among themselves at school and the topics they usually covered during these sessions. The findings revealed that their behaviours are typical functions of their lack of awareness and low utilization of the Adolescent Friendly Health Services initiatives. In that regards, the findings indicated that adolescents are not incorporated in the local Health Facility Committee neither do they partake in designing and development of IEC materials and the distribution thereof and condoms. These trends are contrary to the advocacy by the WHO (2012) which indicates that adolescents should participate in designing, implementation, monitoring and evaluation of their own programmes in order to build adolescent buy-in and to increase the demand for the services. Where necessary, adolescent should advise on needed adjustments, to ensure that their health requirements are met. This in return, creates opportunity to enhance adolescents' ownership of the programme and henceforth, a successful implementation of adolescent programmes.

\subsection{Conclusions}

The findings indicate that the majority of the adolescents as the intended users of adolescent friendly health services initiative did not know about the service. As a result, the majority of the study participants portrayed risky sexual behaviours. Thus the findings inversely answered the study question whether adolescent girls in Otjozondjupa region are aware of the Adolescent Friendly Health Services.

\subsection{Recommendations}

Based on the findings regarding the "active participation" component of the adolescents in the Adolescent Friendly Health Service initiative, the study recommended multi-sectoral innovation of diverse sources of the Information, Education and Communication for the deliverance of health messages to the adolescents. One such innovation is the introduction of on-line health information services and social networking system for the adolescents in order to increase adolescent audience. This approach minimizes the discomfort that is associated with face-to-face information sessions but rather as the literature claims, the approach promotes learning and retention by the adolescents because young people prefer to learn about sensitive issues on their own, using written or audio-visual materials (Pathfinder International, 2002). Moreover, deliverance of messages of adolescent-targeted services should target places where adolescents spend most of their time, like schools and the sport events in order to increase the adolescents' utilization of the services.

Equally, the basic education system should intensify the offering of the extra-curricular life skill training for the learners, as articulated in the manual for extra-curricular life skill training. This in return would create awareness of reproductive related issues, which in return, would guide the adolescents to seek services regarding reproductive health at the health facilities.

\subsection{Study Delimitation and Limitation}

The study was delimited to the school attendee teenage girls only and non-school attendee teenage girls were excluded. Therefore, the findings on this component cannot be generalized to all teenage girls in the region. Moreover, and given the sensitive perspective of the study, the participants may have given limited information which may have influenced the findings.

\section{Acknowledgements}

Acknowledgement is accorded to the Ministry of Health and Social Services and the Ministry of Education for the approvals of the study, granting the prime researcher the permission to collect the data in the health facilities and from learners at different schools in Otjozondjupa region respectively.

The respondents are sincere acknowledged for their cooperation and for providing the information which are necessary for the improvement of deliverance of the Adolescent Friendly Health Services initiatives.

\section{Competing Interests Statement}

The authors declare that there is no conflict of interest. 


\section{References}

Brink, H., van Rensburg, G., \& van der Walt, C. (2012). Fundamentals of Research Methodology for Health Care Professionals (2nd ed.). Cape Town: Juta \& Co. (Pty) Ltd.

Berer, M. (2013). Young people, sex and relationships: miles to go and promises to keep. Editorial.

De Vos, A., Strydom, H., Fouche, C., \& Delport, C. (2011). Research at Grass Roots: For the Social Sciences and human service professions (4th ed.). Pretoria: Van Schaik.

Directorate Education Otjozondjupa Regional Council. (2014). Statistics of Adolescent girls in Schools within Otjozondjupa RegionNamibia: Windhoek.

Larke, L., \& Cleophas-Mazige, B. (2010). Impact of the MEMA kwaVijana Adolescent Sexual and Reproductive Health Interventions on Use of Health Services by Young People in Rural Mwanza, Tanzania: Results of a Cluster Randomized Trial. Journal of Adolescent Health, 47(1010), 512-522. https://doi.org/10.1016/j.jadohealth.2010.03.020

Ministry of Health and Social Services. (2011). Namibia, National Standards for Adolescent Friendly Health Services. MoHSS: Windhoek.

Ministry of Health and Social Services. (2012). Report on the 2012 National HIV Sentinel Survey. Namibia: Windhoek.

Ministry of Health and Social Services Otjozondjupa Regional Office. (2014). Health Facilities and Number of Nurses in Otjozondjupa Region. Namibia: Otjiwarongo

Muyenga, M. L. (2017). Assessment of the implementation by nurses of the Adolescent Friendly.

Muyenga, M. L., Amakali, K., \& Wilkinson, W. (2017) Deliverance of the Adolescent Friendly Health Service standards by nurses in Otjozondjupa Region of Namibia. Global Journal of Health Science, 9(8).

National Rural Health Mission of India. (2006). Implementation Guide on Reproductive and Child. Health II: Adolescent Reproductive Sexual Health Strategy for State and District Programme Managers. New Delhi; India.

Njoroge, K., Olsson, P., Pertet, A. M., \& Ahlberg, B. M. (2010). Voices unheard: Youth and sexuality in the wake of HIV prevention in Kenya. Sexual and Reproductive Healthcare, 1(4) 143-148. https://doi.org/10.1016/j.srhc.2010.07.006

Parahoo, K. (2006). Nursing Research: Principle, Process and Issues (6th ed.). New York: Palgrave MacMillan.

Pathfinder International. (2002). Clinic Assessment of Youth Friendly Services: A Tool for Assessing and Improving Reproductive Health Services for Youth. Watertown.

UNICEF (a). (2011). My future is my choice: Extra-curricular life skills training manual for adolescents"Protecting our peers from HIV infection". Geneva: WHO.

United Nations High Commission for Refugees, United Nations Population Fund. (2012). Save the Children: Adolescent Sexual and Reproductive Health Programs in Humanitarian Settings. Washington DC: UN.

World Health Organisation. (2002). Adolescent friendly health services: An agenda for change. Geneva: WHO.

World Health Organisation. (2006). Defining sexual health Report of a technical consultation on sexual health. Geneva: WHO.

World Health Organization. (2008). Department of Child and Adolescent Health and Development: Orientation Programme on Adolescent Health for Health-Care Providers, Facilitator's Guide. Geneva: United Nation.

World Health Organization. (2012). Making Health Services Adolescent Friendly: Developing National Quality Standards for Adolescent Friendly Health Services. Geneva: WHO.

\section{Copyrights}

Copyright for this article is retained by the author(s), with first publication rights granted to the journal.

This is an open-access article distributed under the terms and conditions of the Creative Commons Attribution license (http://creativecommons.org/licenses/by/4.0/). 